\title{
High-Performance Wavelength-Locked Diode Lasers
}

\author{
Paul Leisher*, Kirk Price, Scott Karlsen, David Balsley, Doug Newman, \\ Rob Martinsen and Steve Patterson \\ nLIGHT Corp, 5408 NE 88th Ste, Bldg E, Vancouver, WA, USA 98665
}

\begin{abstract}
Rapidly maturing industrial laser applications are placing ever-tighter constraints on spectral width and wavelength emission stability over varying operating temperatures of high power diode laser pump sources. For example, improved power scaling and efficiency can be achieved by pumping the narrow upper laser level of Nd:YAG solid state lasers at $885 \mathrm{~nm}$ and the 1532-nm absorption band of Er:YAG solid state lasers, though taking full advantage of these configurations requires wavelength-locked pump sources. nLIGHT offers a wide variety of wavelength-locked diode products based on external volume grating optics technology. It is often believed that the use of external gratings to wavelength lock diode lasers leads to an unavoidable loss in power and efficiency. nLIGHT's design methodology is shown to eliminate the problem in our grating-locked diode laser products. These results are expected to enable improved performance in diode-pumped solid state and fiber laser systems.
\end{abstract}

Keywords: Diode laser, semiconductor laser, narrow linewidth, wavelength locking, Bragg grating, Er:YAG, Nd:YAG

\section{MOTIVATION}

Rapidly maturing industrial laser applications are placing ever-tighter constraints on spectral width and wavelengthtemperature control of high power diode laser pump sources. For example, improved power scaling and efficiency can be achieved by pumping the narrow upper laser level of Nd:YAG solid state lasers at $885 \mathrm{~nm}$ and the 1532-nm absorption band of Er:YAG solid state lasers, though taking full advantage of these configurations requires diode pump sources with narrowed spectral lines [1,2]. Figure 1 illustrates the absorption spectra of Nd:YAG and Er:YAG at these wavelengths. As shown, the absorption feature at $885 \mathrm{~nm}$ in Nd:YAG is $<2.5 \mathrm{~nm}$ FWHM and at $1532 \mathrm{~nm}$ in Er:YAG is $<1 \mathrm{~nm}$ FWHM. The typical unlocked $885 \mathrm{~nm}$ broad area diode laser spectral width is $\sim 2.5 \mathrm{~nm}$, with the peak wavelength shifting at $\sim 0.25 \mathrm{~nm} /{ }^{\circ} \mathrm{C}$; at 1532 -nm the spectral width of the diode increases to $\sim 6 \mathrm{~nm}$ with the peak shifting at $\sim 0.4 \mathrm{~nm} /{ }^{\circ} \mathrm{C}$. Efficient laser systems requiring uniform absorption of the pump light require the diode source be wellmatched (in terms of spectral width and spectral position) to the absorption feature. The drift of operating wavelength with temperature of conventional diode lasers also sets strict requirements on thermal control of the diode pump source.
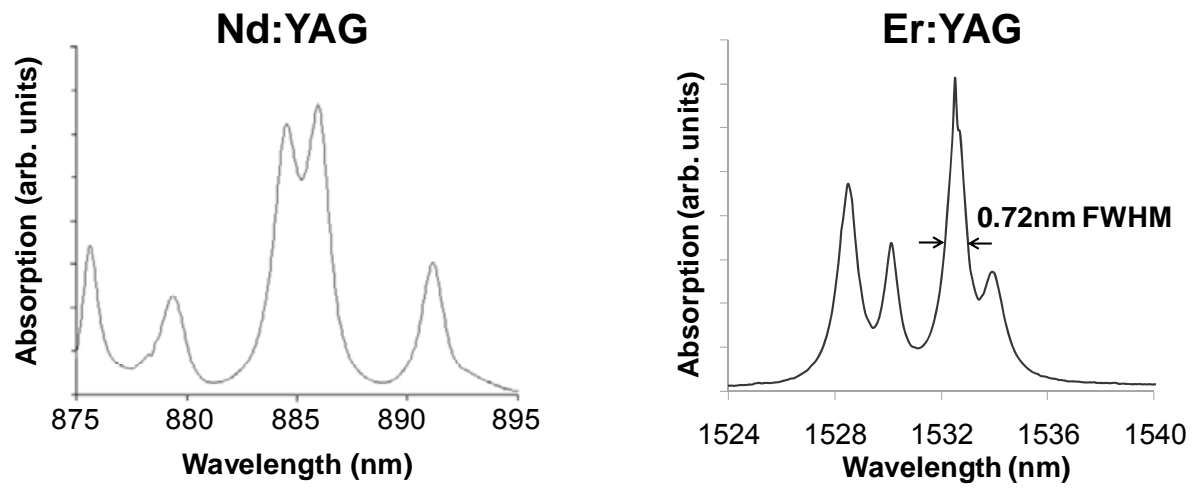

Fig. 1: The absorption spectra of (left) Nd:YAG around 885-nm [1] and (right) Er:YAG around 1532nm [2].

*paul.leisher@nLIGHT.net; phone 360.713.5230; http://www.nLIGHT.net

Sponsorship by the High Energy Laser Joint Technology Office (HEL-JTO), Albuquerque, NM is acknowledged 
In this work, we detail nLIGHT's progress in the development and commercialization of wavelength-locked high-power diode lasers, with results presented at $885-\mathrm{nm}$ and $1532-\mathrm{nm}$. Leveraging our expertise in precision optical alignment, our approach utilizes external feedback by means of volumetric Bragg grating optics. Our approach provides a number of advantages over other methods of wavelength locking. One such benefit is wavelength flexibility - while the results here are specific to just two wavelengths, the approach itself is wavelength agnostic, spanning the full gamut of nLIGHT's laser diode product family (from 630-nm to 2100-nm). Furthermore, we demonstrate that when properly implemented, the approach provides excellent line narrowing and stabilization of peak operating wavelength over temperature with virtually no loss in power or efficiency relative to unlocked pump performance.

\section{VHG LOCKED DIODE LASERS}

The optical lasing spectra of quantum well semiconductor lasers are closely related to the optical gain spectrum of the active region. Standard broad area diode lasers do not employ wavelength-selective feedback or longitudinal mode suppression mechanisms, resulting in lasing on any and all modes which experience sufficient round-trip gain within the spectral gain bandwidth of the active region. The envelope spectral width of the lasing modes is therefore predominantly dependent on the spectral width of the optical gain, which is itself proportional to the square of the emission wavelength [3]. In other words, spectral width and wavelength drift with temperature cannot be directly engineered through epitaxy modifications alone in a given material system.

Line-narrowing and wavelength stabilization of diode lasers is typically achieved through wavelength-selective feedback which locks the laser to one (or a few) longitudinal modes. The two most common approaches utilized in the high power diode laser arena are internal buried distributed Bragg gratings (DFBs) [4] and external volumetric Bragg gratings (also commonly referred to as VHGs for volume holographic grating) [5-6]. VHGs are external optical components in which a periodic variation in index of refraction has been written into the photorefractive glass, which thereby provides wavelength- (and angular-) selective feedback. This optic is then carefully aligned to the output beam of the diode, locking the wavelength of the diode laser (by effectively forming an extended cavity laser having wavelength-selective feedback). This is illustrated schematically in Figure 2.

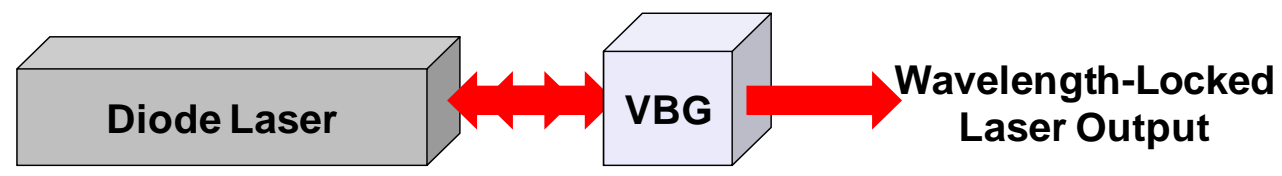

Fig. 2: Schematic illustration of external wavelength locking with a VHG. Note optimization must now be considered for the extended cavity laser resonator.

nLIGHT has standardized on this approach (rather than internal DFB gratings) for the following reasons:

1. Flexibility - Linewidths can be tailored for the application of interest. Wavelength changes are easy, requiring only a change in the locking optic. There is no need to grow new material, pattern and then etch gratings for each minor change in wavelength requested by the customer.

2. Improved yield and reliability, lower cost - External locking eliminates the need for complex epitaxy regrowth methods typically employed in buried grating approaches. Alignment leverages the same welldeveloped techniques used in lensing. Unlocked and locked products can be built from chips of the same wafer.

3. Better temperature stability - The locking optic (having a naturally low $\partial \lambda / \partial \mathrm{T}$ ) can be thermally decoupled from the laser diode, to the degree the glass absorbs energy and shifts by $\partial \mathrm{n} / \partial \mathrm{T}$, further improving temperature stability relative to internal grating approaches.

4. Highest power and efficiency - The epitaxy can be independently optimized for high power and high efficiency, without the need to compromise the design for good grating coupling to the optical mode required by buried grating approaches. The low internal loss of the grating itself allows design optimizations which provide zero penalty in the power and efficiency relative to the unlocked design this is shown in the next section. 
Copyright 2009 Society of Photo-Optical Instrumentation Engineers.

This paper was published in the proceedings of the SPIE Photonics West 2009, Vol. 7198-38 (2009), High-Power Diode Laser Technology and Applications VII, and is made available as an electronic reprint with permission of SPIE. One print or electronic copy may be made for personal use only. Systematic or multiple reproduction, distribution to multiple locations via electronic or other means, duplication of any material in this paper for a fee or for commercial purposes, or modification of the content of the paper are prohibited.

\section{RESULTS}

High power cm-bars on microchannel-coolers may be collimated in the fast axis and vertically stacked. Figure 3 (a) and (b) illustrate the performance of a VHG-locked 6-bar stack operating $\mathrm{cw}$ at $25^{\circ} \mathrm{C}$ with a wavelength around $885 \mathrm{~nm}$ [7]. At a rated current of $110 \mathrm{~A}$, the locked unit produces $585 \mathrm{~W}$ of power at $45 \%$ conversion efficiency, (representing $<2.5 \%$

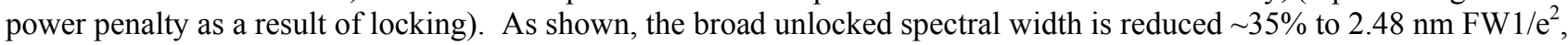
and while still quite broad relative to narrow linewidths typically demonstrated by locking methods, it is still well-suited for pumping the $885 \mathrm{~nm}$ Nd:YAG absorption feature. More recently, making use of a higher-efficiency epitaxy design [7], a VHG-locked 7-bar stack operating $\mathrm{cw}$ at $25^{\circ} \mathrm{C}$ was demonstrated, producing $>680 \mathrm{~W}$ of power at $54 \%$ conversion

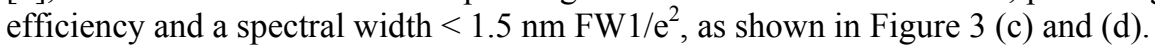

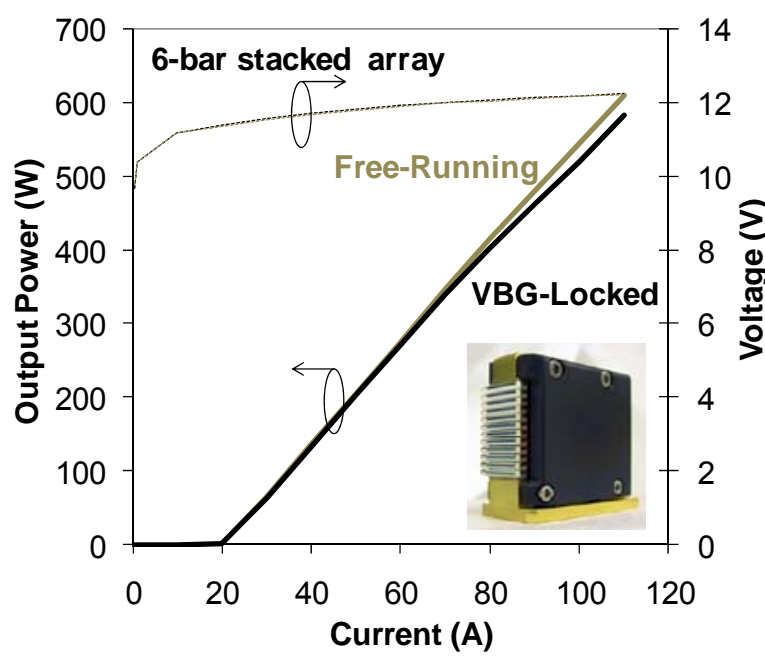

(a)

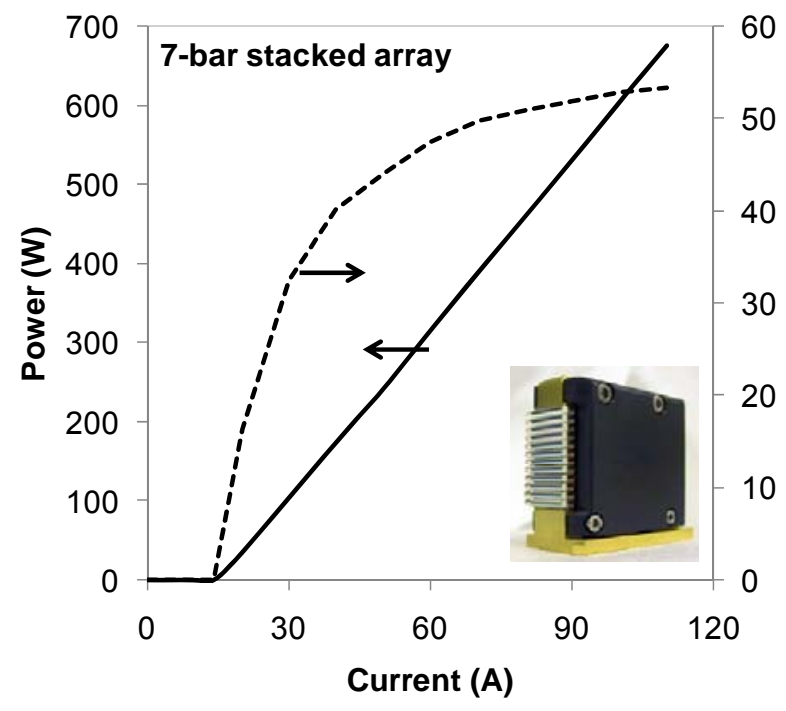

(c)

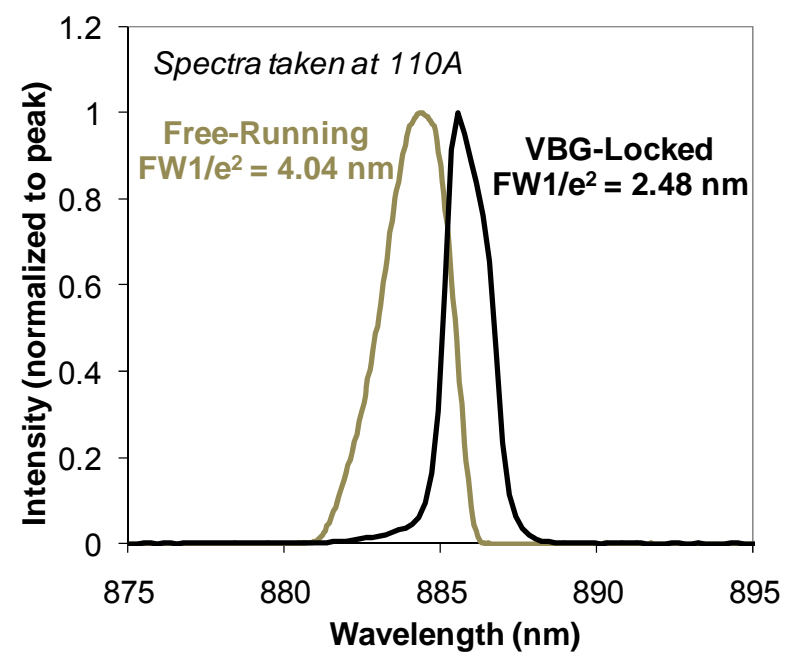

(b)

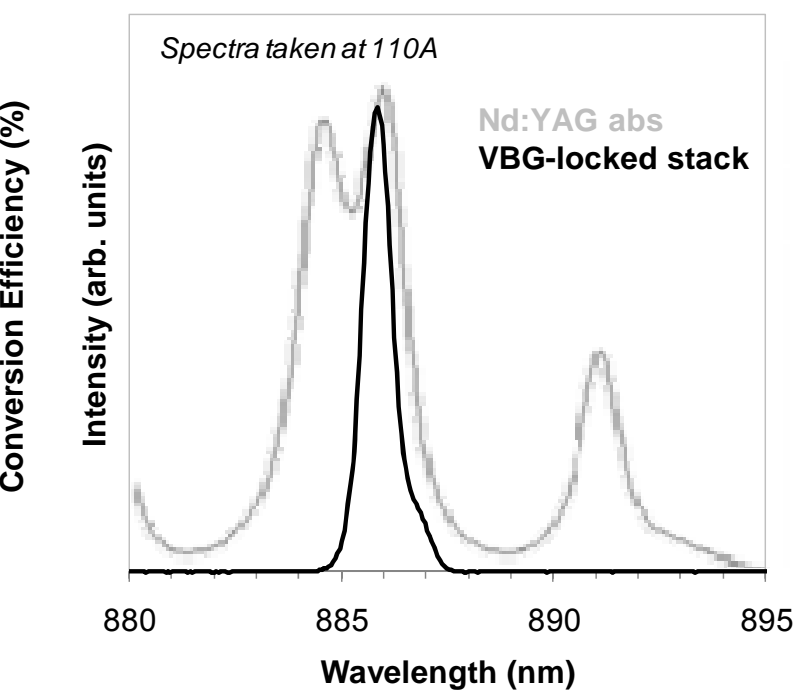

(d)

Fig. 3: (a and b) Power vs. current and spectra for a 6-bar stacked array operating around $885 \mathrm{~nm}$. As shown, the VBG reduces the laser linewidth by $\sim 35 \%$ with $<5 \%$ power penalty [7]. (c and d) Recent advancements in the laser epitaxy have improved power conversion efficiency of locked stacks. This 7-bar stack produces $>680 \mathrm{~W}, 54 \% \mathrm{E} / \mathrm{O}$ at the rated $110 \mathrm{~A}, 25^{\circ} \mathrm{C}$ operating conditions. Further optimizations to nLIGHT's grating design approach are expected to enable modules operating at $>60 \%$ conversion efficiency. 
Copyright 2009 Society of Photo-Optical Instrumentation Engineers.

This paper was published in the proceedings of the SPIE Photonics West 2009, Vol. 7198-38 (2009), High-Power Diode Laser Technology and Applications VII, and is made available as an electronic reprint with permission of SPIE. One print or electronic copy may be made for personal use only. Systematic or multiple reproduction, distribution to multiple locations via electronic or other means, duplication of any material in this paper for a fee or for commercial purposes, or modification of the content of the paper are prohibited.

To meet the brightness needs of end-pumped solid state and fiber lasers, nLIGHT offers a conductively-cooled fiber coupled package solution (Pearl ${ }^{\mathrm{TM}}$ ) based on arrays of singles emitters [8-9]. The inset of Figure 4 depicts an optical photograph of one such package. Figure 4 illustrates the light and efficiency curves, as well as optical lasing spectra, of a $60 \mathrm{~W}$ (rated) 1532-nm Pearl module operating $\mathrm{cw}$ at $15{ }^{\circ} \mathrm{C}$, both before and after VBG-locking. The unit was measured afocal (fiber-coupled results unavailable at the time of publication); typical fiber coupling optical-to-optical efficiencies coupling to a $400 \mu \mathrm{m}$ core $0.22 \mathrm{NA}$ fiber in this configuration are 95 to $98 \%$. As shown, nLIGHT's wavelength locking approach narrows the emissions spectrum to $<0.2 \mathrm{~nm}$, stabilizing it at $1532 \mathrm{~nm}$ with a $17 \mathrm{~dB}$ side mode suppression ratio (SMSR) at the rated 12A operating current. It is expected that the SMSR will be further improved to beyond $20 \mathrm{~dB}$ in the next three months. The power and efficiency are unchanged as a result of locking. Figure 5 compares the unlocked and locked lasing spectra to the absorption spectrum of Er:YAG around 1532-nm. This result demonstrates that through careful optimization, very narrow spectral widths $(<0.2 \mathrm{~nm})$ with good locking (high SMSR) can be achieved with no penalty to laser power or efficiency.

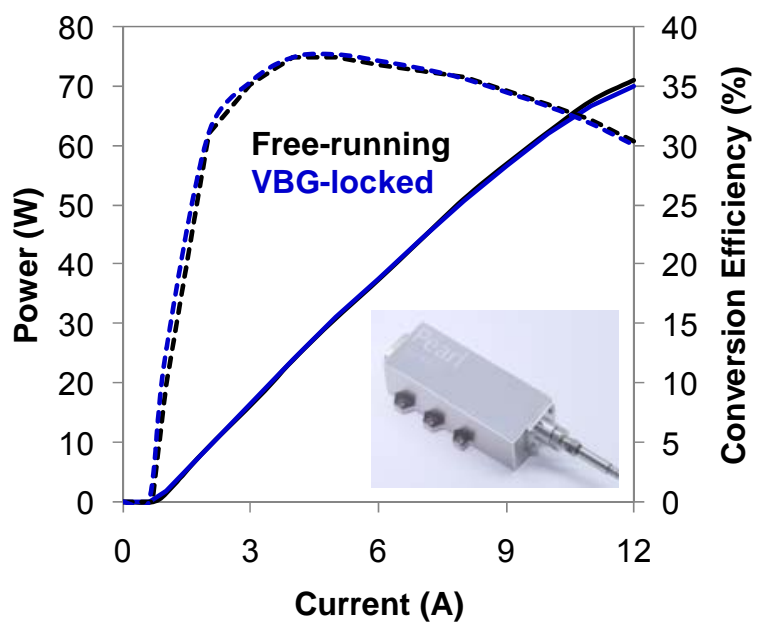

(a)

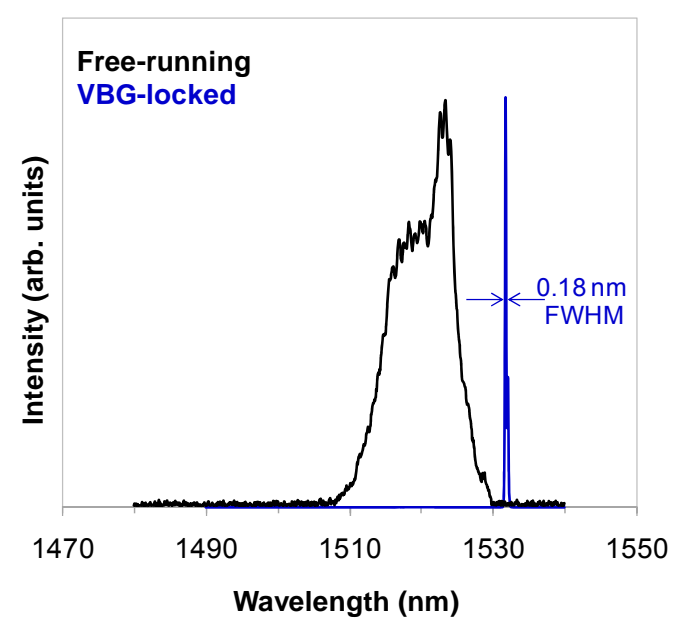

(b)

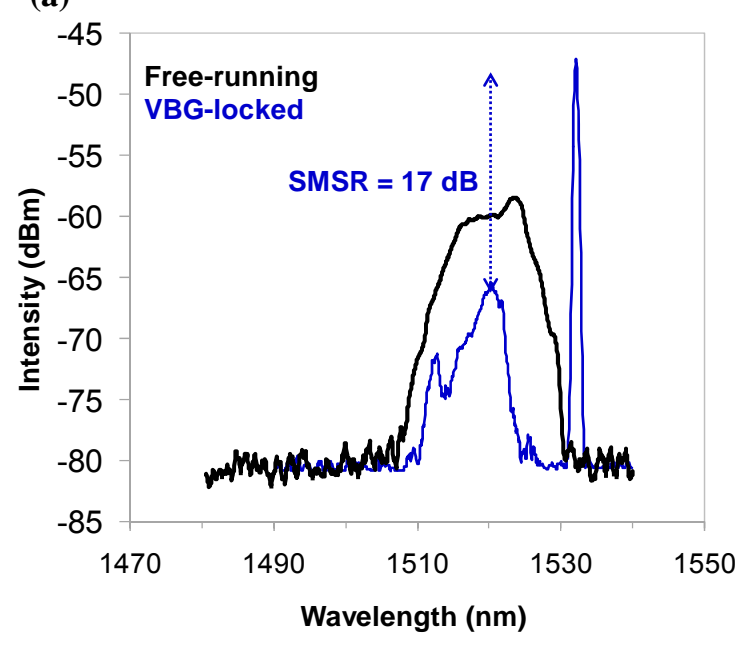

(c)

Fig. 4: (a) Power and efficiency versus drive current for a $15 x x-n m$ Pearl module before and after VBG locking. This unit is measured afocal and demonstrates $>70 \mathrm{~W}$ peak power and $>37 \%$ peak conversion efficiency. Fiber coupled results were not available at the time of publication (optical to optical coupling efficiency to a $400 \mu \mathrm{m}$ core, $0.22 \mathrm{NA}$ fiber is typically 95 to 98\%). (Bottom) The optical lasing spectrum (taken at 12A) before and after VBG-locking shown on a linear (bottom left) and $\log$ (bottom right) scale. The wavelength is narrowed to $<0.2 \mathrm{~nm}$ FWHM and locked to 1532 $\mathrm{nm}$ with a $17 \mathrm{~dB}$ side-mode suppression ratio (SMSR). As shown, the unit suffers no performance penalty as a result of wavelength stabilization. 


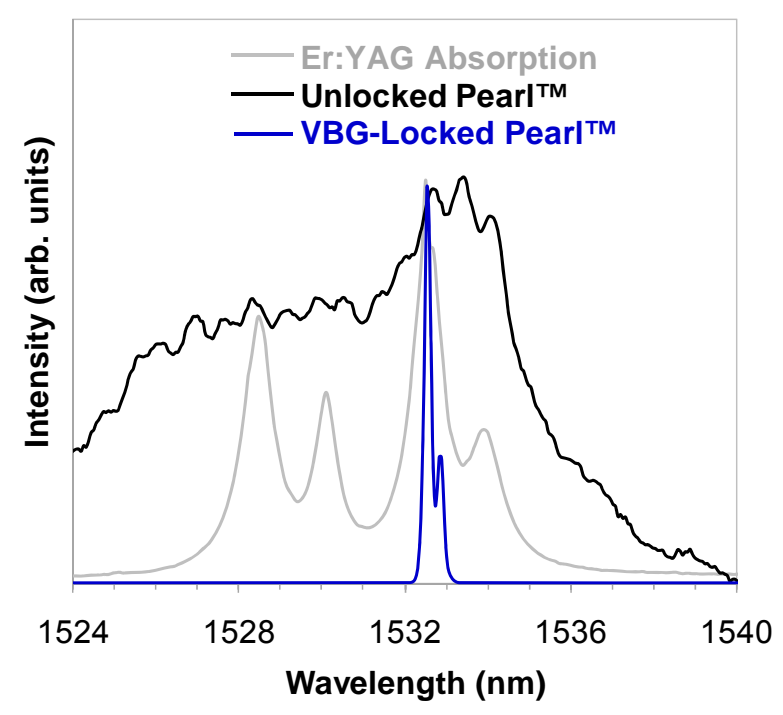

Fig. 5: Comparison of the unlocked and locked spectra with the absorption spectrum of Er:YAG around $1532 \mathrm{~nm}$. The unlocked and locked spectra for the prototype unit were shifted on the plot by $10-\mathrm{nm}$ and $0.72-\mathrm{nm}$, respectively, to align with the peak absorption feature around 1532-nm. (In practice, this wavelength shift could be easily accomplished with a slight perturbation to the VHG design itself or by temperature tuning [10]).

\section{CONCLUSION}

Applications such as direct pumping of Nd:YAG at 885-nm and of Er:YAG at 1532-nm are placing ever-increasing demands on diode pump linewidth and temperature stability. To meet these requirements, some method of wavelengthselective feedback must be employed. nLIGHT offers variety of wavelength locked products (single emitters, microchannel-cooled bars, stacks, and fiber-coupled modules) based on external locking with volumetric Bragg gratings (VBGs/VHGs). Commercial VBG-locked 885-nm microchannel-cooled bars now operate at 100W/bar with $>54 \%$ conversion efficiency and $<1 \mathrm{~nm}$ FWHM spectral width. At 1532-nm, a VBG-locked Pearl ${ }^{\mathrm{TM}}$ module demonstrates $>70 \mathrm{~W}$ peak power and $>37 \%$ peak efficiency with $<0.20 \mathrm{~nm}$ FWHM spectral width. nLIGHT's external locking approach is wavelength agnostic and is believed to offer the highest possible power and efficiency of any wavelengthlocking scheme: the diode epitaxy can be independently optimized for high power / high efficiency unlocked operation and emitters subsequently locked with an external grating with virtually no penalty to power and conversion efficiency.

\section{REFERENCES}

[1] M. Frede, et al., Optics Lett., vol. 31, pp. 3618-3619, (2006).

[2] S. D. Setzler, et al., IEEE J. Sel. Top. Quant. Elect., vol. 11, no. 3, pp. 645-657, (2005).

[3] S.M. Sze, Physics of Semiconductor Devices, Wiley, New York, 2nd ed. (1981).

[4] J. Carroll, et al., Distributed Feedback Semiconductor Lasers, IEE/SPIE, London, (1998).

[5] G. Steckman, et al., IEEE J. Sel. Top. Quant. Elect., vol. 13, no. 3, pp. 672-678, (2007).

[6] L. Glebov, Photonics Spectra, Jan. (2005).

[7] P. Leisher, et al., Proc. SPIE, vol. 6952, (2008).

[8] S. Patterson, et al., DEPS $20^{\text {th }}$ SSDLTR, (2007).

[9] http://www.nLIGHT.net/diodes/family/1 Pearl-HighPower-FiberCoupled-Modules

[10] T. Crum, et al, Proc. SSDLTR, pp. 84-86, (2008). 